\title{
Review of: "Evaluating Human Mutation Databases for 'Treatability' Using Personalized Antisense Oligonucleotides"
}

John Rossi ${ }^{1}$

1 City of Hope

Potential competing interests: The author(s) declared that no potential competing interests exist.

This survey/review of a bottoms up approach to identifying rare genetic diseases that are potentially treatable with ASO technologies is quite valuable to the antisense community and should be published without further revision. 